\title{
Infectious Complications With Nondaily Versus Daily Infusion of Intravenous Fat Emulsions in Non-Critically Ill Adults
}

\author{
Mary Lou Chheng, PharmD $^{1}$; Charles Heidbreder, PharmD $^{1}$; \\ Imad F. Btaiche, PharmD, BCNSP ${ }^{2}$; and Allison Beck Blackmer, PharmD, BCPS ${ }^{1}$
}

\begin{abstract}
Background: Increased risk for infection has been associated with the administration of intravenous fat emulsion (IVFE). Typically, IVFE is infused daily as part of the parenteral nutrition (PN) regimen. However, a national IVFE shortage in 2010 compelled institutions to restrict administration to nondaily. This retrospective study evaluated the rate of infections associated with the nondaily as compared to daily IVFE infusion in hospitalized adult patients. Methods: Patients in the study group received nondaily IVFE during the shortage period, and patients in the control group received daily IVFE. The primary outcomes were the development of catheter-related bloodstream infections (CR-BSIs) or any bloodstream infection (BSI). Secondary outcomes were the development of respiratory, urinary, wound, or other infections. Results: Included in the study were 52 patients, 33 patients in the study group and 19 patients in the control group. There were no CR-BSIs reported. BSIs occurred in 1 patient in the study group. The total number of infections and urinary tract infections (UTIs) per 1000 catheter days were not different between the 2 groups ( 45.28 vs $21.24, P=.203)$ and $(24.39$ vs $5.525, P=.099)$, respectively. Survival analyses showed no difference between the 2 groups for the time to first infection (11.24 vs 6.59 days, $P=.30$ ) and time to first UTI ( 11.97 vs 7 days, $P=.093$ ), respectively. Conclusions: Nondaily vs daily IVFE infusion did not have a significant effect on the risk of infection or time to development of infection; however, results are limited due to the small sample size. Large prospective randomized clinical trials are needed to further evaluate the effect of daily as compared to nondaily IVFE infusion on infectious complications. (Nutr Clin Pract. 2013;28:737-744)
\end{abstract}

\section{Keywords}

parenteral nutrition; intravenous fat emulsion; infection

Parenteral nutrition (PN) is a standard therapy for hospitalized patients who are malnourished or at risk for malnutrition and are unable to meet nutrition requirements via the oral or enteral route. Hospitalized patients who receive $\mathrm{PN}$ are at increased risk of infection, and the use of intravenous fat emulsion (IVFE) may be an additional risk factor for infectious complications. ${ }^{1-4}$ IVFEs are used in PN as a source of calories and essential fatty acids. They are also precursors of eicosanoids that affect platelet aggregation, immune function, and inflammation. ${ }^{3-5}$ The use of IVFE, however, may be associated with a potential increase in infectious complications, especially bloodstream infections. ${ }^{1,3,4}$ Currently available IVFEs in the United States are made from soybean oil that supplies long-chain triglycerides (LCTs). ${ }^{3,4,6}$ The slightly alkaline, isotonic IVFE solution provides a favorable milieu for microbial growth including grampositive, gram-negative, and fungal microorganisms. ${ }^{3,7,8}$ Based on in vitro studies that have shown prolific microbial growth in IVFE, the Centers for Disease Control (CDC) has recommended a maximum IVFE infusion time of no longer than 12 hours. ${ }^{3,8,9}$ Furthermore, LCTs in IVFEs may have immunosuppressive effects, therefore concerns have been raised regarding the possibility of increased infection risk with IVFE infusion. ${ }^{3}$ It has been postulated that the reticuloendothelial system (RES) function may become impaired when high amounts of infused
IVFE compete with microorganisms for clearance and LCT deposition in the liver diminishes the ability of Kupffer cells to sequester bacteria. ${ }^{3,6,10,11}$ IVFE may also interfere with neutrophil and macrophage function thereby decreasing the ability to fight infections. ${ }^{4}$ Despite these concerns, there is a lack of clear data to support the role IVFE plays with respect to infectious risk. ${ }^{12-15}$ Whereas some clinical studies reported a higher incidence of infections in patients who received PN with IVFE as compared to PN without IVFE, although the difference was not statistically significant, ${ }^{15-16}$ others reported that IVFE may possibly reduce the occurrence and severity of infections by improving the activity of lymphocytes and cytokines. ${ }^{17-18}$

From ${ }^{1}$ The University of Michigan College of Pharmacy, Ann Arbor, Michigan; and ${ }^{2}$ Lebanese American University, School of Pharmacy, Byblos, Lebanon.

Financial disclosure: None declared.

This article originally appeared online on November 5, 2013.

Corresponding Author:

Allison Beck Blackmer, PharmD, BCPS, Department of Clinical, Social and Administrative Sciences, University of Michigan College of Pharmacy, 428 Church St, Ann Arbor, MI 48109, USA.

Email: ablackm@umich.edu. 
Table 1. Definitions: Immunosuppressive Conditions and Agents.

\begin{tabular}{lll}
\hline Immunosuppressive Conditions & & \multicolumn{1}{c}{ Immunosuppressive Agents } \\
\hline - Cancer and/or receiving chemotherapy & - Corticosteroids \\
- Transplantation & - Calcineurin inhibitors (eg, cyclosporine, tacrolimus) \\
- HIV/AIDS & - Antimetabolic agents (eg, methotrexate, sirolimus, azathioprine, mercaptopurine) \\
- Systemic lupus erythematosus & - Biological agents (eg, daclizumab, infliximab, etanercept, vincristine, vinblastine, \\
- Rheumatoid arthritis & & \\
- Psoriasis & & \\
- Systemic bacterial/fungal infection &
\end{tabular}

The standard practice is to administer IVFE daily with PN, unless it is otherwise contraindicated or not required to meet caloric needs. A national shortage of IVFE in 2010-2011 compelled healthcare institutions to conserve IVFE supplies by providing IVFE less frequently. ${ }^{19}$ At our institution, patients who weighed $\geq 30 \mathrm{~kg}$ received IVFEs twice weekly during the shortage period. To date, no published studies have evaluated the rate of infectious complications in patients who receive daily IVFE as compared to nondaily/intermittent IVFE infusions. The aim of this study was to retrospectively evaluate the effects of nondaily vs daily IVFE infusion on the development of infectious complications in hospitalized adult patients who received IVFE as part of their PN therapy.

\section{Methods}

This was a retrospective evaluation of the effects of nondaily vs daily IVFE infusion on infectious complications in hospitalized adult patients at a tertiary care health system. Hospitalized patients over 18 years of age who weighed at least $30 \mathrm{~kg}$ and received PN with at least 1 dose of IVFE via a peripherally inserted central catheter (PICC) during the period between May 19, 2010, and August 31, 2011, were included in the study. The study group included patients who received IVFE twice weekly (nondaily IVFE) during the period of IVFE shortage, between May 19, 2010, and March 30,2011 . The control group included patients who received daily IVFE following the end of the shortage between March 31, 2011, and August 31, 2011. Patients were excluded if 1 or more of the following criteria were met: pregnant females; received $\mathrm{PN}$ prior to hospital admission; located in or transferred from an intensive care unit (ICU); received propofol, clevidipine, or liposomal amphotericin B; received antibiotics or antifungals for longer than 48 hours prior to PN initiation; received antibiotics or antifungals during PN therapy without documented infection; were immunosuppressed or received immunosuppressive agents (Table 1); received PN through an intravenous (IV) catheter other than a PICC; received cycled ( $<24$ hours) IVFE infusion; received blood transfusions; had severe liver disease (Table 2); and/or were treated with dialysis.
Patients were identified using electronic patient medical records and pharmacy data systems. Collected data included demographics (age, gender, race, weight, and height); hospital length of stay; indication for PN; duration of IV catheter placement before PN or IVFE initiation; IV catheter type; number of catheter lumens; signs of infection (body temperature over $38^{\circ} \mathrm{C}$, elevated white blood cell count, and absolute neutrophil count); serum glucose concentrations; total daily calories from PN; daily IVFE dose (ml); and the duration of IVFE treatment. Microbiology data included all positive cultures including specimen type, microbiological organism, and reported sensitivities for positive cultures. In addition, antimicrobial regimens were documented for each positive culture (Table 2).

The primary outcomes were the development of catheterrelated bloodstream infections (CR-BSIs) or any bloodstream infection (BSI). Secondary outcomes included the development of pneumonia, urinary tract infections (UTIs), wound infections, the composite of any other infection, and the time to development of first infection. The study was approved by the Institutional Review Board.

\section{Statistics}

A previously published study in which infectious complications were evaluated when IVFE was withheld for the first 7-10 days of PN in surgical ICU patients provided the basis for the power analysis. ${ }^{6}$ A sample size of 253 patients in each group was deemed necessary to detect a difference in incidence of infectious complications between the 2 groups, with $80 \%$ power and a 2-sided significance level of .05. Data were analyzed from the first day of PN initiation and up to 30 days or until patient discharge from the hospital, whichever came first. Descriptive statistics were used for comparisons between the 2 groups. Student's $t$ test, Wilcoxon rank sums and negative binomial distribution were used for continuous data analysis. Chi-square and Fisher's exact tests were used for analysis of categorical variables. Time to first infection was analyzed using the Kaplan-Meier survival analysis. A regression analysis of survival data was performed to evaluate the effects of variables on hazard rates. SAS software (SAS Institute Inc, Cary, NC, USA) was used for data analysis. 
Table 2. Pertinent Study Definitions.

\begin{tabular}{|c|c|}
\hline Term & Definition \\
\hline Severe liver diseases & $\begin{array}{l}\text { - Documented hepatitis } \\
\text { - Documented alcohol and drug induced liver disease } \\
\text { - Documented cirrhosis } \\
\text { Clinically significant elevations in LFTs as follows: } \\
\text { Increased serum alanine aminotransferase (ALT; normal range 7-35 IU/L), aspartate } \\
\text { aminotransferase (AST; normal range } 8-30 \mathrm{IU} / \mathrm{L} \text { ), alkaline phosphatase (ALP; normal range } \\
\text { 30-130 IU/L) concentrations at least } 3 \text { times the upper limit of normal in patients with previously } \\
\text { normal values, OR } \\
\text { If baseline (at least } 2 \text { measurements performed during the current hospital period) serum } \\
\text { ALT, AST, or ALP concentrations were elevated, then increased serum ALT, AST, or ALP } \\
\text { concentrations at least } 3 \text { times higher than baseline concentrations OR } \\
\text { Any increased serum ALT, AST, or ALP concentrations associated with increased total bilirubin } \\
\text { (normal range } 0.2-1.2 \mathrm{mg} / \mathrm{dL}) \geq 2.5 \mathrm{mg} / \mathrm{dL}\end{array}$ \\
\hline $\begin{array}{l}\text { Catheter-related bloodstream } \\
\text { infection (CR-BSI) }\end{array}$ & $\begin{array}{l}\text { - Positive intravenous catheter tip cultures or a positive blood culture drawn from the catheter consistent with } \\
\text { a simultaneously drawn peripheral blood culture and a duration of antibiotic therapy for at least } 10 \text { days }\end{array}$ \\
\hline Bloodstream infection & - Positive blood culture and a duration of antibiotic therapy for at least 10 days \\
\hline Wound infection & $\begin{array}{l}\text { - Positive culture from the surgical site or a positive pelvic fluid culture and a duration of antibiotic } \\
\text { therapy for at least } 7 \text { days }\end{array}$ \\
\hline Respiratory tract infection & - Positive sputum culture and a duration of antibiotic therapy for at least 7 days \\
\hline Urinary tract infection & - Positive urine culture and duration of antibiotic therapy for at least 3 days \\
\hline Intra-abdominal infection & $\begin{array}{l}\text { Positive peritoneal fluid culture or a positive abdominal abscess culture and a duration of antibiotic } \\
\text { therapy for at least } 10 \text { days }\end{array}$ \\
\hline "Other" infections & $\begin{array}{l}\text { - Positive cultures from "other" sites managed with appropriate antibiotics for an appropriate length of } \\
\text { time }\end{array}$ \\
\hline
\end{tabular}

LFT, liver function tests.

\section{Results}

A total of 612 patients were evaluated of whom 52 patients met the inclusion criteria. These included 33 patients in the study group who received nondaily IVFE, and 19 patients in the control group who received daily IVFE. Of those excluded, 306 patients met at least 1 exclusion criteria and the other 254 patients met at least 2 exclusion criteria. The most common reason for exclusion was the administration of antibiotics for longer than 48 hours prior to PN initiation (Table 3).

Overall baseline characteristics were similar between the 2 groups (Table 4) with the following exceptions: patients in the study group had significantly fewer IVFE treatment days (1.48 vs 6.47 days, $P<.0001)$ and more catheter days prior to IVFE initiation (1.85 vs 0.53 days, $P=.0016)$, as compared to the control group, respectively. The mean number of catheter lumens was also significantly higher in the study group as compared to the control group (2.27 vs 1.94 , respectively, $P=.034$ ). Nutrition data were similar between the 2 groups (Table 5), although the study group received significantly lower average daily lipid calories as compared to patients in the control group, $3.68 \mathrm{vs} 4.96 \mathrm{kcal} / \mathrm{kg} /$ day respectively, $P=.012$.

BSIs occurred in 1 patient in the study group (Table 6). There were no CR-BSIs. Overall, 14 patients (26.9\%) had documented infections, including 10 patients in the study group $(30.3 \%)$ and 4 patients in the control group $(21.1 \%)$ who developed at least 1 type of infection. Of all infections, 76.5\% occurred in the study group as compared to $23.5 \%$ in the control group. Eight patients developed UTIs, of whom 7 patients were in the study group. One patient in the control group developed pneumonia.

The number of infections per 1000 catheter days was not significantly different between the study and control groups (45.28 vs 21.24 , respectively, $P=.203$ ). The number of UTIs per 1000 catheter days was also not significantly different between the study and control groups (24.39 vs 5.525, respectively, $P=.099$ ).

Survival analyses for time to first infection (11.24 vs 6.59 days, $P=.30$ ) (Figure 1) and time to first UTI (11.97 vs 7 days, $P=.093$ ) (Figure 2) showed no difference between the study and control groups, respectively. However, there was a trend toward significance for time to first UTI. Average serum glucose concentrations, time to IVFE initiation, and mean IVFE calories did not have an effect on infectious complications and were not shown to be predictors of infection $(P=.56, .72$, and .87 , respectively) for either group.

Survival analysis for time to any infection in relation to the number of catheter lumens showed no statistically significant difference when accounting for nondaily vs daily IVFE (HR = $2.086,95 \% \mathrm{CI}=0.0671-6.483, P=.204)$. When all infections were considered in aggregate $(\mathrm{HR}=2.338,95 \% \mathrm{CI}=0.811$ $6.742, P=.116$ ) (Figure 3). Similarly, there was no significant 
Table 3. Number of Patients Meeting Study Exclusion Criteria.

\begin{tabular}{lc}
\hline Exclusion Criterion & $\begin{array}{c}\text { Number of } \\
\text { Patients }\end{array}$ \\
\hline Antibiotic > 48 hrs prior to PN initiation & 271 \\
Immunosuppressive agents & 119 \\
Cycled IVFE & 93 \\
Blood transfusion within 48 hrs of PN or during PN & 89 \\
Antifungal > 48 hrs prior to PN initiation & 70 \\
Chemotherapy & 53 \\
<1 dose of IVFE & 31 \\
Receipt of antibiotic during PN therapy without & 23 \\
$\quad$ documented infection & 20 \\
Propofol, clevidipine, or liposomal amphotericin B & 19 \\
Severe liver disease/encephalopathy & 17 \\
Transferred from ICU & 12 \\
Dialysis & 11 \\
PN prior to hospital admission & 10 \\
Patient transferred from outside hospital with other & \\
$\quad$ exclusion criteria & 9 \\
Catheter other than PICC & 6 \\
No patient records & 6 \\
PICC placed at outside hospital & 6 \\
<18 years old & 4 \\
Located in ICU & \\
Antifungal during PN therapy without documented & 2 \\
Pregnant & 4 \\
Prisoner & \\
\hline
\end{tabular}

ICU, intensive care unit; IVFE, intravenous fat emulsion; PICC, peripherally inserted central catheter; $\mathrm{PN}$, parenteral nutrition. Out of 612 patients identified, 560 were excluded from the study. Study subjects may have met $>1$ exclusion criterion. Thus, the numbers depicted do not total the number of subjects excluded.

difference in the time to UTI in relation to the number of catheter lumens when accounting for nondaily vs daily IVFE (HR $=2.982,95 \% \mathrm{CI}=0.685-12.99, P=.146)$ (Figure 4). However, there was a trend toward significance when considering all UTIs $(\mathrm{HR}=3.657,95 \% \mathrm{CI}=0.864-15.479, P=.078)$.

\section{Discussion}

The use of IVFE may be associated with a potential increase in infectious complications, especially BSIs, although it remains unclear whether infections are associated with PN infusion or more specifically IVFE. ${ }^{1,3,4,15,16}$

In this study, the frequency of IVFE administration did not statistically significantly increase the risk of infectious complications or the time to first infection. BSIs were observed in 1 patient in the study group with a corresponding overall BSI rate of $0.02 \%$. The low incidence of BSI is in contrast to data from a study that evaluated the impact of delaying IVFE administration for 7-10 days in 64 surgical ICU patients where CR-BSIs and BSIs were reported in $29.6 \%$ of patients. ${ }^{6}$ The higher incidence of CR-BSIs and BSIs in the latter study may be a reflection of the inclusion of critically ill patients who are at higher risk for infection as compared to our study that excluded critically ill patients, and the stringent criteria used for patient inclusion in our study.

In this study, no significant differences in pneumonia or CR-BSIs were found. Clinical studies of surgical patients who received IVFE reported increased risk of infectious complications including pneumonia, UTIs, and wound infections. ${ }^{6,16,20,21}$ A study utilizing immunological data showed that the capacity of neutrophils to eradicate Streptococcus pneumoniae was negatively impacted by the presence of IVFE. ${ }^{21}$ In a prospective study of 60 trauma patients, patients were randomized to receive $\mathrm{PN}$ therapy complete with IVFE or to PN therapy with IVFE withheld for the first 10 days of therapy; a significantly higher rate of pneumonia and line sepsis in patients who received IVFE was observed. ${ }^{20}$ One possible explanation for the observed differences between previous studies and our study may be that patients in our study received IVFE infused over 24 hours. Clinical studies of soybean-oil-based IVFE have shown that intermittent 10-hour IVFE infusion for 3 days decreases RES function by $40 \%$ whereas a continuous infusion did not negatively affect RES function. ${ }^{3,11}$ Considering the possible numerous mechanisms by which IVFE may increase the risk of infection, one may speculate that the lower infection rate observed in our study may be theoretically a reflection of the 24 hour IVFE infusions with possibly lesser impact on RES function. ${ }^{6,11,20,22}$

Although our study results showed no difference for the time to first UTI between the 2 groups, the rate of UTIs was higher in patients who received nondaily IVFE as compared to daily IVFE. Four of the 8 patients who developed a UTI were males who had bladder cancer and were admitted to the surgery urology service for radical cystectomy. Three of those 4 patients were in the study group. It is possible that the occurrence of UTI was associated with the patients' urological conditions and may not necessarily relate to IVFE infusion. In a prospective study of 140 patients who underwent urological interventions that included patients with benign prostate hyperplasia, bladder cancer, prostate cancer, and urolithiasis, the rate of UTI was reported at $21.4 \% .^{23}$ Because patients undergoing urological manipulations are at high risk of developing nosocomial UTIs, patients in our study may have developed a UTI as a result of urology surgery regardless of IVFE infusion.

The higher rate of infection observed in the study group, although not significant, may be attributed to the higher number of intravenous catheter lumens. Although some studies have shown that single-lumen catheters are associated with a lower risk of infection than multilumen catheters, this observation has not been consistent in all studies. ${ }^{2,24-26}$ A prospective 
Table 4. Patient Demographics, Parenteral Nutrition Indicators, Catheter Data, \& Clinical Indicators of Infection.

\begin{tabular}{|c|c|c|c|}
\hline Demographic & Nondaily IVFE (Study Group, n=33) & Daily IVFE (Control Group, $\mathrm{n}=19$ ) & $P$ Value \\
\hline \multicolumn{4}{|l|}{ Gender } \\
\hline Female & $\mathrm{n}=14(42.42 \%)$ & $\mathrm{n}=11(57.89 \%)$ & \\
\hline Male & $\mathrm{n}=19(57.58 \%)$ & $\mathrm{n}=8(42.11 \%)$ & .28 \\
\hline \multicolumn{4}{|l|}{ Race } \\
\hline Caucasian & $\mathrm{n}=28(84.85 \%)$ & $\mathrm{n}=18(94.74 \%)$ & \\
\hline African American & $\mathrm{n}=5(15.15 \%)$ & $\mathrm{n}=1(5.26 \%)$ & .39 \\
\hline Age (yrs) & $55.12 \pm 16.35$ & $62.17 \pm 12.14$ & \\
\hline Mean \pm SD [range] & [21-85] & [33-85] & .11 \\
\hline Height $(\mathrm{cm})$ & $170.86 \pm 10.66$ & $168.1 \pm 11.05$ & \\
\hline Mean \pm SD [range] & {$[142.24-193]$} & [149.4-189] & .38 \\
\hline Weight $(\mathrm{kg})$ & 77.1 & 82.7 & .38 \\
\hline Median [range] & {$[47-162.39]$} & [48.8-168.9] & \\
\hline $\operatorname{BMI}\left(\mathrm{kg} / \mathrm{m}^{2}\right)$ & 25.65 & 29.6 & .12 \\
\hline Median [range] & {$[17.94-57.81]$} & {$[17.12-48.28]$} & \\
\hline Percentage with BMI $\left(\mathrm{kg} / \mathrm{m}^{2}\right)>30$ & 30.3 & 42.11 & .39 \\
\hline Hospital Length of Stay (days) & 14 & 13 & \\
\hline Median [range] & {$[5-25]$} & {$[4-29]$} & .86 \\
\hline \multicolumn{4}{|l|}{ Indication for PN } \\
\hline NPO & $\mathrm{n}=14(42.42 \%)$ & $\mathrm{n}=10(52.63 \%)$ & \\
\hline Malnutrition & $\mathrm{n}=5(15.15 \%)$ & $\mathrm{n}=3(15.79 \%)$ & \\
\hline Ileus & $\mathrm{n}=5(15.15 \%)$ & $\mathrm{n}=3(15.79 \%)$ & \\
\hline $\mathrm{SBO}$ & $\mathrm{n}=3(9.09 \%)$ & $\mathrm{n}=0$ & \\
\hline Fistula & $\mathrm{n}=3(9.09 \%)$ & $\mathrm{n}=0$ & \\
\hline Wound Infection & $\mathrm{n}=1(3.03 \%)$ & $\mathrm{n}=0$ & \\
\hline GI bleed & $\mathrm{n}=1(3.03 \%)$ & $\mathrm{n}=0$ & \\
\hline Elevated pancreatic enzymes & $\mathrm{n}=1(3.03 \%)$ & $\mathrm{n}=1(5.26 \%)$ & .489 \\
\hline Surgery & $\mathrm{n}=0$ & $\mathrm{n}=2(10.53 \%)$ & \\
\hline Number PN days & $5 \pm 3.03$ & $6.79 \pm 6.01$ & \\
\hline Mean \pm SD [range] & [2-19] & {$[1-27]$} & .087 \\
\hline Number IVFE days & $1.48 \pm 0.75$ & $6.47 \pm 3.84$ & \\
\hline Mean \pm SD [range] & {$[1-4]$} & {$[2-15]$} & $<.0001$ \\
\hline Number of catheter lumens & $2.27 \pm 0.52$ & $1.94 \pm 0.52$ & \\
\hline Mean \pm SD [range] & {$[1-3]$} & {$[1-3]$} & .034 \\
\hline Number catheter days & $8.69 \pm 5.44$ & $9.52 \pm 6.74$ & \\
\hline Mean \pm SD [range] & [3-32] & [3-29] & .58 \\
\hline Catheter days prior to $\mathrm{PN}$ initiation & $0.61 \pm 0.9$ & $0.47 \pm 0.84$ & \\
\hline Mean \pm SD [range] & {$[0-3]$} & {$[0-3]$} & .59 \\
\hline Catheter days prior to IVFE initiation & $1.85 \pm 1.87$ & $0.53 \pm 0.84$ & \\
\hline Mean \pm SD [range] & {$[0-7]$} & {$[0-3]$} & .0016 \\
\hline Mean WBC count (k/mm3) & $8.57 \pm 2.69$ & $9.3 \pm 3.27$ & .39 \\
\hline Mean \pm SD [range] & {$[5.42-14.53]$} & {$[5.1-16.3]$} & \\
\hline
\end{tabular}

BMI, body mass index; GI, gastrointestinal; IVFE, intravenous fat emulsion; NPO, nil per os; PN, parenteral nutrition; SBO, small bowel obstruction; WBC, white blood cell; yrs, years. Catheter refers to central intravenous catheter (e.g. PICC line).

surveillance study reported that each additional lumen significantly increased the risk of CR-BSI by about 4.4 times $(\mathrm{HR}=$ 3.3, $P<.001) .{ }^{26}$ A meta-analysis reported that multilumen catheters were associated with only slightly higher risk of infection as compared to single-lumen catheters and concluded that multilumen catheters do not pose a significant risk factor for increased risk of BSIs. ${ }^{24}$ Although the impact of catheter lumens on the rate of BSI remains debatable, the CDC guidelines recommend using catheters with the lowest number of ports and lumens possible. ${ }^{2}$

\section{Limitations}

This study presents with limitations that include its retrospective design and small sample size. Although the study did not meet the predefined power calculation, the sample size is 
Table 5. Nutrition Data.

\begin{tabular}{|c|c|c|c|}
\hline Criterion & Nondaily IVFE (Study Group, $\mathrm{n}=33$ ) & Daily IVFE (Control Group, $\mathrm{n}=19$ ) & $P$ Value \\
\hline $\begin{array}{l}\text { Mean total kcal/kg/day } \\
\text { Mean } \pm \mathrm{SD} \text { [range] }\end{array}$ & $\begin{array}{c}20.79 \pm 6.04 \\
{[9.47-33]}\end{array}$ & $\begin{array}{l}24.22 \pm 5.95 \\
{[13.13-39.85]}\end{array}$ & .0523 \\
\hline $\begin{array}{l}\text { Mean PN kcal } / \mathrm{kg} / \text { day } \\
\text { Mean } \pm \mathrm{SD} \text { [range] }\end{array}$ & $\begin{array}{c}17.2 \pm 4.96 \\
{[8.23-25.94]}\end{array}$ & $\begin{array}{l}19.26 \pm 4.66 \\
{[10.24-30.4]}\end{array}$ & .13 \\
\hline $\begin{array}{l}\text { Mean lipid kcal } / \mathrm{kg} / \text { day } \\
\text { Mean } \pm \mathrm{SD}[\mathrm{range}]\end{array}$ & $\begin{array}{l}3.68 \pm 1.78 \\
{[0.89-8.38]}\end{array}$ & $\begin{array}{c}4.96 \pm 1.6 \\
{[2.77-9.45]}\end{array}$ & .012 \\
\hline $\begin{array}{l}\text { Mean serum glucose while on PN }(\mathrm{mg} / \mathrm{dL}) \\
\text { Mean } \pm \mathrm{SD} \text { [range] }\end{array}$ & $\begin{array}{c}126.7 \pm 26.8 \\
{[100.9-206.3]}\end{array}$ & $\begin{array}{l}119.2 \pm 25.39 \\
{[91.75-203.3]}\end{array}$ & .33 \\
\hline
\end{tabular}

IVFE, intravenous fat emulsion; PN, parenteral nutrition.

Table 6. Infection Rate.

\begin{tabular}{|c|c|c|c|}
\hline Criterion & Nondaily IVFE (Study Group, n=33) & Daily IVFE (Control Group, $\mathrm{n}=19$ ) & $P$ Value \\
\hline \multicolumn{4}{|l|}{ Primary outcomes } \\
\hline Catheter-related bloodstream infection & 0 & 0 & - \\
\hline Bloodstream infection & $1(8 \%)$ & 0 & - \\
\hline \multicolumn{4}{|l|}{ Secondary outcomes } \\
\hline Pneumonia & 0 & $1(25 \%)$ & - \\
\hline Urinary tract infection & $7(54 \%)$ & $1(25 \%)$ & - \\
\hline Wound infection & $3(23 \%)$ & $2(50 \%)$ & - \\
\hline Abdominal infection & $2(15 \%)$ & 0 & - \\
\hline Total \# of infections & 13 (76.5\% of all infections) & $4(23.5 \%$ of all infections $)$ & - \\
\hline \# of patients with infections & $10(30.3 \%)$ & $4(21.1 \%)$ & \\
\hline \# of infections per 1000 catheter days & $45.28 \pm 14.06$ & $21.24 \pm 11.44$ & .203 \\
\hline Mean \pm SD [range] & {$[24.64-83.20]$} & {$[7.39-61.06]$} & \\
\hline \# of UTI per 1000 catheter days & $24.39 \pm 9.22$ & $5.53 \pm 5.53$ & .099 \\
\hline Mean \pm SD [range] & {$[11.63-51.16]$} & {$[0.78-39.22]$} & \\
\hline
\end{tabular}

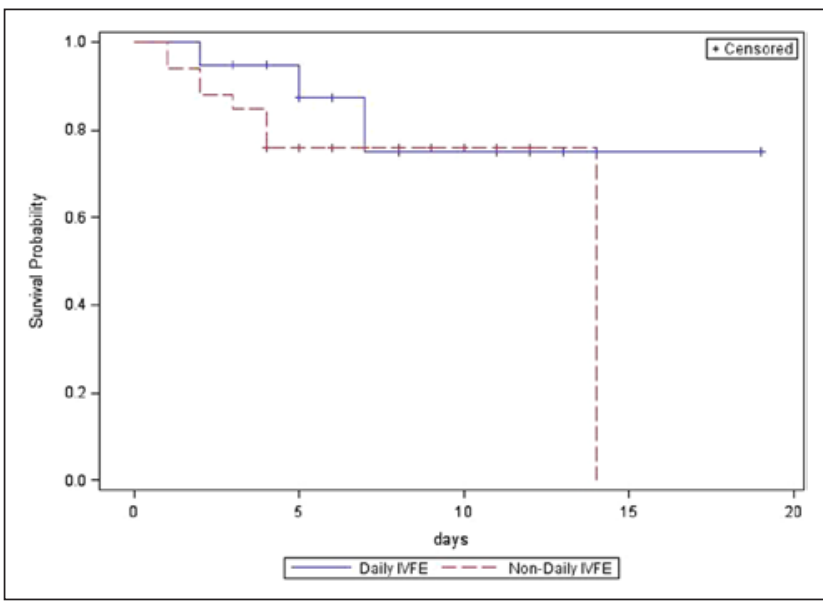

Figure 1. Survival analysis for time to first infection: Daily intravenous fat emulsion (IVFE) vs nondaily IVFE. The mean number of days for the nondaily IVFE group was 11.24 days, as compared to 6.59 days for the daily IVFE group, $P=.3$.

however comparable to those of other similar studies. ${ }^{6,16,20}$ The indication for PN therapy is included in Table 4. While the groups appear comparable, due to the large variety of indications

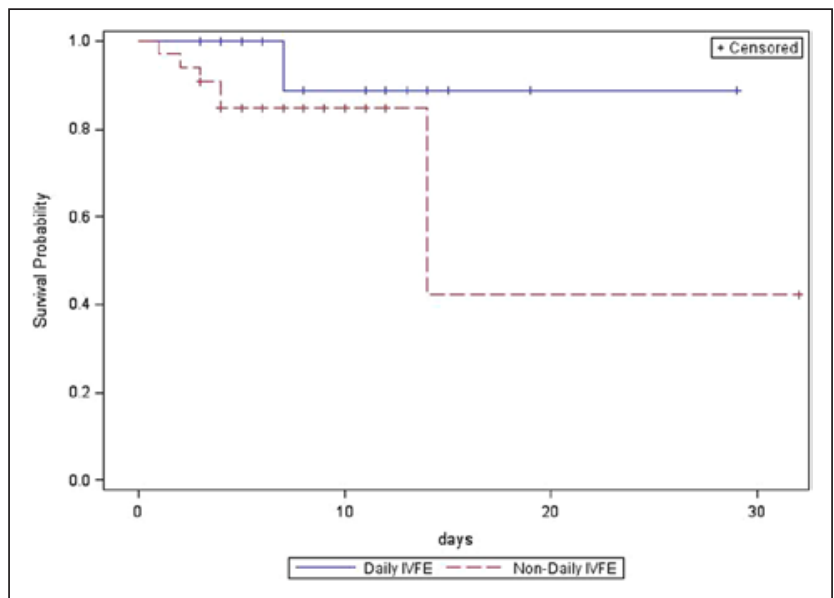

Figure 2. Survival analysis for time to first urinary tract infection (UTI): Daily intravenous fat emulsion (IVFE) vs nondaily IVFE. The mean number of days was 11.97 in the nondaily group, as compared to 7 days in the daily IVFE group, $P=.093$.

and small sample sizes within each group, calculating significance between the groups was not possible. It remains possible that differences between the groups exist, which is another 


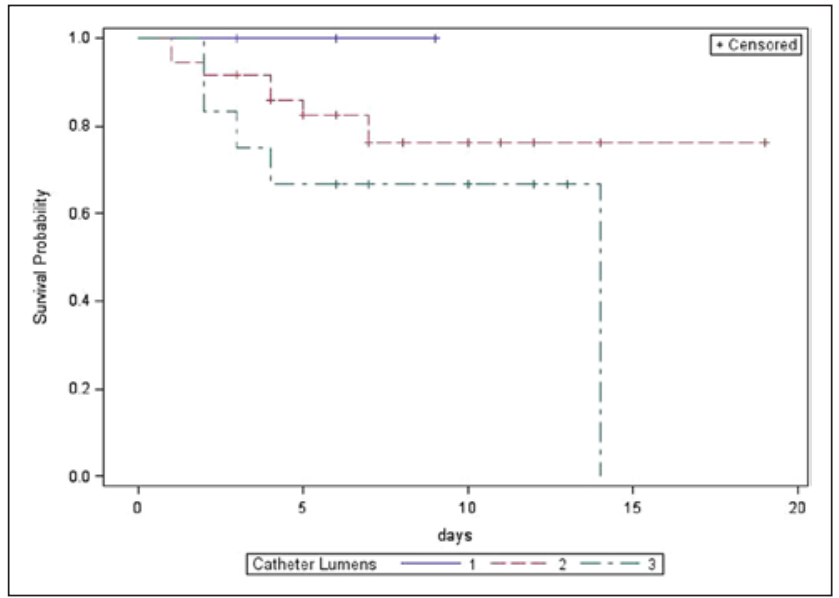

Figure 3. Survival analysis for time to any infection vs number of catheter lumens. $\mathrm{HR}=2.338,95 \% \mathrm{CI}=0.811-6.742, P=.116$.

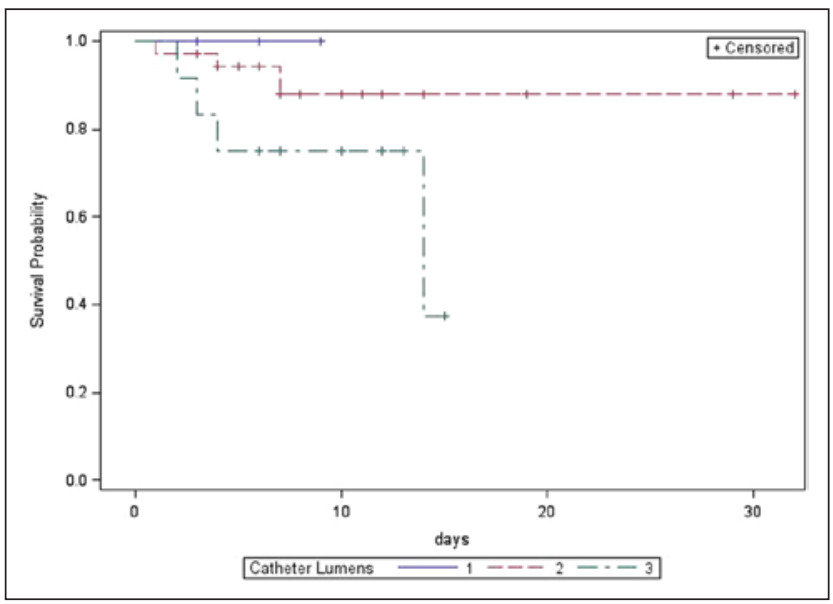

Figure 4. Survival analysis for time to urinary tract infection (UTI) vs number of catheter lumens. $\mathrm{HR}=2.982,95 \% \mathrm{CI}=$ $0.685-12.99, P=.146$.

limitation to be considered. The strict inclusion criteria in this study can be viewed as a strength and limitation. Although the stringent inclusion criteria minimized the effects of confounders on study results, they limited the sample size and reduced the external validity of the study as results may not necessarily be generalized to a larger population.

\section{Conclusion}

Nondaily vs daily IVFE infusion did not have a significant effect on the rate of infectious complications or the time to development of infection. Large prospective clinical studies are needed to further evaluate the impact of daily vs nondaily IVFE infusion on infectious complications.

\section{Acknowledgments}

We are grateful to Kathleen B. Welch, MS, MPH, at the Center for Statistical Consultation and Research at the University of Michigan in Ann Arbor, Michigan, for her assistance with statistical analysis.

\section{Author's Note}

At the time of the study, Dr. Chheng and Dr. Heidbreder were fourth year pharmacy students at the University of Michigan College of Pharmacy.

\section{References}

1. Btaiche IF, Khalidi N, Kovacevich DS, eds. The University of Michigan Hospitals and Health Centers Parenteral and Enteral Nutrition Manual. Ann Arbor: University of Michigan; 2010.

2. O'Grady NP, Alexander M, Burns LA, et al. Guidelines for the prevention of intravascular catheter-related infections. Centers for Disease and Control Prevention. CID. May 2011;52:1-32.

3. Warshawsky KY. Intravenous fat emulsions in clinical practice. Nutr Clin Pract. 1992;7(4):187-196.

4. Mirtallo JM, Dasta JF, Kleinschmidt KC, et al. State of the art review: intravenous fat emulsions: current applications, safety profile, and clinical implications. Ann Pharmacother. 2010;44(4):688-700.

5. Kruger PS. Forget glucose: what about lipids in critical illness? Crit Care Resusc. 2009;11(4):305-309.

6. Gerlach AT, Thomas S, Murphy CV, et al. Does delaying early intravenous fat emulsion during parenteral nutrition reduce infections during critical illness? Surg Infect. 2011;12(1):43-47.

7. Melly MA, Meng HC, Schaffner W. Microbiol growth in lipid emulsions used in parenteral nutrition. Arch Surg. 1975;110(12):1479-1481.

8. Gilbert M, Gallagher SC, Eads M, et al. Microbial growth patterns in a total parenteral nutrition formulation containing lipid emulsion. J Parenter Enteral Nutr. 1986;10(5):494-497.

9. Baker MT, Naguib M. Propofol: the challenges of formulation Anesthesiology. 2005;103(4):860-876.

10. Jensen GL, Mascioli EA, Seidner DL, et al. Parenteral infusion of longand medium-chain triglycerides and reticuloendothelial system function in man. J Parenter Enteral Nutr. 1990;14(5):467-471.

11. Seidner DL, Mascioli EA, Istfan NW, et al. Effects of long-chain triglyceride emulsions on reticuloendothelial system function in humans. J Parenter Enteral Nutr. 1989;13(6):614-619.

12. Sihler KC, Chenoweth C, Zalewski C, et al. Catheter-related vs. catheterassociated blood stream infections in the intensive care unit: incidence, microbiology, and implications. Surg Infect (Larchmt). 2010;11(6):529-534.

13. Byrnes MC, Coopersmith CM. Prevention of catheter-related blood stream infection. Curr Opin Crit Care. 2007;13(4):411-415.

14. Freeman J, Goldmann DA, Smith NE, et al. Association of intravenous lipid emulsion and coagulase-negative staphylococcal bacteremia in neonatal intensive care units. $N$ Engl J Med. 1990;323(5):301-308.

15. Avila-Figueroa C, Goldmann DA, Richardson DK, et al. Intravenous lipid emulsions are the major determinant of coagulase-negative staphylococcal bacteremia in very low birth weight newborns. Pediatr Infect Dis $J$. 1998;17(1):10-17.

16. McCowen KC, Friel C, Sternberg J, et al. Hypocaloric total parenteral nutrition: effectiveness in prevention of hyperglycemia and infectious complications: a randomized clinical trial. Crit Care Med. 2000;28(11): 3606-3611.

17. Hasselmann M, Reimund JM. Lipids in the nutritional support of the critically ill patients. Curr Opin Crit Care. 2004;10(6):449-455. 
18. Monson JR, Ramsden CW, MacFie J, Brennan TG, Guillou PJ. Immunorestorative effect of lipid emulsions during total parenteral nutrition. Br J Surg. 1986;73(10):843-846.

19. Vanek VW, Allen P, Harvey Banchik LP, et al. Information to use in the event of an intravenous fat emulsion shortage. A.S.P.E.N.; 2011 Available at: www.nutritioncare.org. Accessed April 26, 2011.

20. Battistella FD, Widergren JT, Anderson JT, et al. A prospective, randomized trial of intravenous fat emulsion administration in trauma victims requiring total parenteral nutrition. $J$ Trauma. 1997;43(1):52-60.

21. Versleijen MW, Roelofs HM, te Morsche RH, et al. Parenteral lipids impair pneumococcal elimination by human neutrophils. Eur $J$ Clin Invest. 2010;40(8):729-734.

22. O'Grady NP, Alexander M, Dellinger EP, et al. Guidelines for the prevention of intravascular catheter-related infections. Centers for Disease
Control and Prevention. MMWR Recomm Rep. August 9, 2002;51 (RR-10):1-29.

23. Westenfelder M, Rosset K, Pelz K. Development of nosocomial and iatrogenic urinary tract infections (UTI) following urological interventions. A prospective clinical study. Scand J Urol Nephrol Suppl. 1987;104: 59-63.

24. Dezfulian C, Lavelle J, Nallamothu BK, et al. Rates of infection for singlelumen versus multilumen central venous catheters: a meta-analysis. Crit Care Med. 2003;31(9):2385-2390.

25. Zurcher M, Tramer MR, Walder B. Colonization and bloodstream infection with single- versus multi-lumen central venous catheters: a quantitative systematic review. Anest Analg. 2004;99(1):177-182.

26. Casaer MP, Mesotten D, Hermans G, et al. Early versus late parenteral nutrition in critically ill adults. $N$ Engl $J$ Med. 2011;365(6):506-517. 